\title{
Abstract \\ Diagnosis of prostate cancer by trans rectal ultrasound guided prostate biopsy; an observational study
}

\author{
Abeynayake $\mathrm{SD}^{1 *}$, Perera $\mathrm{G}^{1}$, Wickramarachchi $\mathrm{D}^{1}$, Warnakulasuriya $\mathrm{T}^{1}$, Upasena $\mathrm{A}^{1}$ \\ ${ }^{1}$ Colombo North Teaching Hospital, Ragama
}

\begin{abstract}
Introduction

Digital rectal examination (DRE) coupled with the measurement of prostate specific antigen (PSA) is widely used for the early diagnosis and monitoring of prostate cancer. In the case of abnormal DRE and/or suspicious PSA value (over $4 \mathrm{ng} / \mathrm{ml}$ ), trans-rectal ultrasound guided prostate biopsy is adopted. Objective was to correlate the ultrasonic appearance with the biochemical markers and histological findings.

Methodology

This observational study was conducted at the Department of Radiology of Colombo North Teaching Hospital from August 2012 to January 2014. An interviewer administered questionnaire was used to collect data from all patients undergoing biopsy. Demographic data, biochemical markers, ultrasonic appearance and histological findings were recorded. An 18-gauge biopsy needle loaded in a spring-action automatic biopsy device was used to procure six $1.5 \mathrm{~cm}$ prostate biopsy specimens from both lobes.

Results

Data from 194 patients were analyzed. Out of patients 30.9\% (60) had cancer on biopsy; $33.5 \%$ (65) had benign adenomatous hyperplasia(BPH) and 34.5\%(67) had chronic prostatitis. Mean (SD) age positive for malignancy was 72.2 (7.6) years. Men with prostate cancer had mean prostate volume of $39.8 \mathrm{ml}$ and mean PSA level of 102.6/dl. $65 \%$ (39) of malignant patients had focal lesions. Men with non malignant histology had mean prostate volume of $44.7 \mathrm{ml}$ and mean PSA level of $23.8 \mathrm{ng} / \mathrm{dl}$. Ultrasonically focal lesions were identified in $19.7 \%$ (26/132) nonmalignant males. Presence of focal lesions on imaging was significantly associated with malignancy $(p<0.001)$ in histology.

Conclusions

Men with prostate cancer were older, had smaller prostate and had higher PSA levels than men with negative biopsies. There is a significant association between finding focal lesion on ultrasound and being diagnosed as malignancy in histology.
\end{abstract}

Key words: Ultrasound guided; Prostate Biopsy; Prostate Cancer

Copyright: (C) 2015 Abeynayake SD et al. This is an open access article distributed under the Creative Commons Attribution License, which permits unrestricted use, distribution, and reproduction in any medium, provided the original work is properly cited.

* Correspondence : snjabey@hotmail.com

Cite this abstract as: Abeynayake SD, Perera G, Wickramarachchi D, Warnakulasuriya T, Upasena A. Diagnosis of prostate cancer by trans rectal ultrasound guided prostate biopsy; an observational study. Anuradhapura Medical Journal 2015;9 (2Supp):S13.

DOI: http://dx.doi.org/10.4038/amj.v9i2Supp.7562 
